\title{
Wireless Sensing using Vehicle Headlamps for Intelligent Transportation Systems: Proof of Concept
}

\author{
Hisham Abuella ${ }^{1, *}$, Sabit Ekin ${ }^{1}$, Samir Ahmed ${ }^{2}$, Farshad Miramirkhani ${ }^{3}$, Burak Kebapci ${ }^{3}$, and Murat Uysal $^{3}$ \\ ${ }^{1}$ School of Electrical and Computer Engineering, OSU, Stillwater \\ ${ }^{2}$ School of Civil and Environmental Engineering, OSU, Stillwater \\ ${ }^{3}$ Department of Electrical and Electronics Engineering, Ozyegin University, Istanbul
}

\begin{abstract}
Vehicular communication and sensing technologies are mainly based on the conventional radio frequency (RF) or laser technologies. These systems suffer from several issues such as RF interference and poor performance in scenarios where the incidence angle between the speed detector and the vehicle is rapidly varying. Introducing a new sensing technology will add diversity to these systems and enhance the reliability of the real-time data. In this study, we investigate our speed estimation sensing system named "Visible Light Detection and Ranging (ViLDAR)". ViLDAR utilizes visible light sensing technology to measure the variation of the vehicle's headlamp light intensity and estimate the vehicle speed. The measurement settings of the ViLDAR experiments are presented. The preliminary results obtained in the real-world environment/setting are promising when compared to the simulations. Additional measurements using the ViLDAR prototype will be conducted under different conditions and scenarios to further optimize the system.
\end{abstract}

\section{Introduction}

Vehicle to vehicle $(\mathrm{V} 2 \mathrm{~V})$ and vehicle to infrastructure (V2I) sensing and communication technologies have received much attention by ITS researchers and industry in order to improve traffic operations and safety 1 . Most of the wireless $\mathrm{V} 2 \mathrm{~V}$ and $\mathrm{V} 2 \mathrm{I}$ systems use radio frequency (RF) technologies, which are well-established systems and operate at their highest potential. Examples of these systems include the RAdio Detection and Ranging (RADAR) and the Light Detection and Ranging (LiDAR) which determine the location and the speed of vehicles. The theory behind the RADAR and LiDAR systems is that they measure the change in the frequency or the travel time of the reflected RF waves from the targeted vehicle. Both RADAR and LiDAR have some limitations and issues that can make the speed estimations unreliable 2. One of these issues is the narrow beam-width required for accurate speed estimation.

Light emitting diode (LED) headlamps are being widely used by vehicle manufacturers because of their longer life and less power consumption. Many researchers have investigated the use of visible light technology in V2V and V2I sensing and communication $[3,4,5]$. A hybrid system that uses RF-based system and visible light communication (VLC) was introduced by Ucar et al. 5 to perform ITS functions. Cheng et al. compared VLC and RF channel in vehicular communication 7. The authors investigated the use of visible light sensing (VLS) for vehicle speed estimation and proposed the Visible Light Detection and Ranging (ViLDAR) sensing system (patent is pending) 8. ViLDAR utilizes the headlamp's light to calculate vehicle speed based on the change in power received at a photodetector (PD) located alongside the road and convert the power to distance 9. The performance of ViLDAR system was investigated in different road scenarios using computer simulations (the details for computer simulations are provided in 9). ViLDAR outperformed the RADAR/LiDAR system under the assumptions discussed in 9. In addition to speed estimation, ViLDAR has potential useful applications in the "Advanced Vehicle Safety Systems" bundle of the national ITS architecture 10.

This paper presents preliminary results of the field implementation of a prototype ViLDAR system to serve as a proof of concept. The measurement environments and the hardware used in the prototype implementation of the system are described. In Section II, the proposed system model is presented along with the proposed algorithm. The field measurements are presented in Section III. Preliminary conclusions and future work are discussed in the last section.

\section{System model and methodology}

The system model is presented in Fig. 1, where $\theta$ and $d$ are the incidence angle and the lateral distance between the vehicle and the PD, respectively. $R$ and $D$ are the varying longitudinal distance and the actual distance between the vehicle and the PD, respectively, at a given instant. The PD collects the measurements, then an analog to digital converter (ADC) digitizes the readings and sends the data to the digital signal processing (DSP) unit to estimate vehicle speed.

* Corresponding author: hisham.abuella@okstate.edu 
The field experiments were conducted on a local road east of the Stillwater regional airport, in Stillwater, Oklahoma, as shown in Error! Reference source not found.. This road was chosen because of its low traffic volume and the ability to close it to traffic if needed to control the measurement environment.

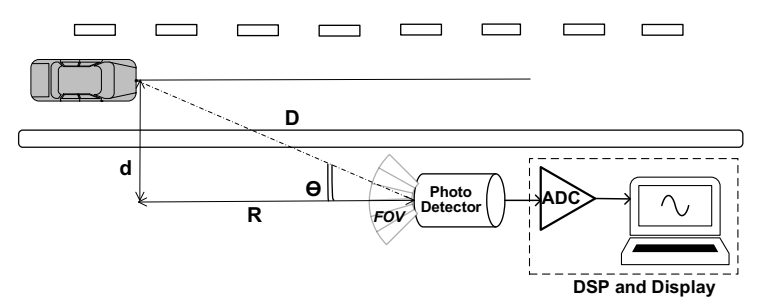

Fig. 1. Prototype system model.

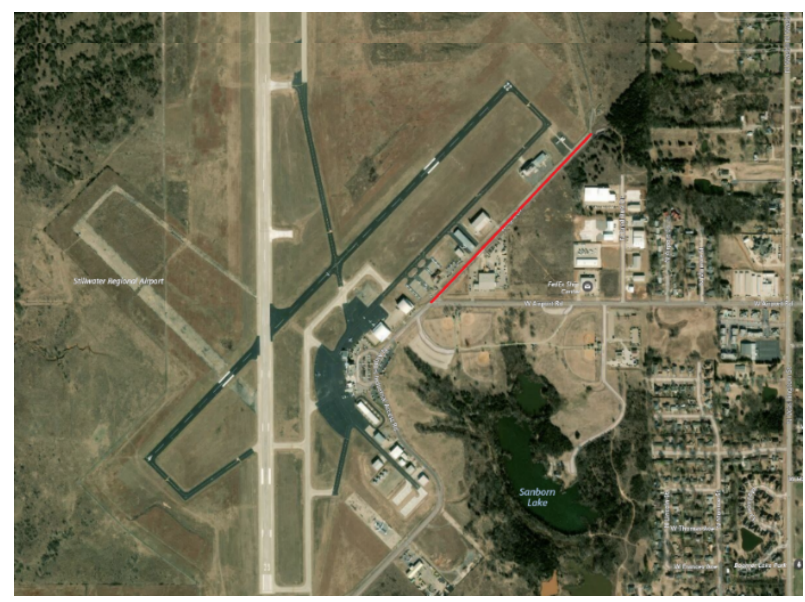

Fig. 2. Experimental site.

Fig. 2 shows photographs of (a) ViLDAR system prototype that includes RaspberryPi, Photodetector and processing unit, and (b) the vehicle approaching the ViLDAR system during the measurements.

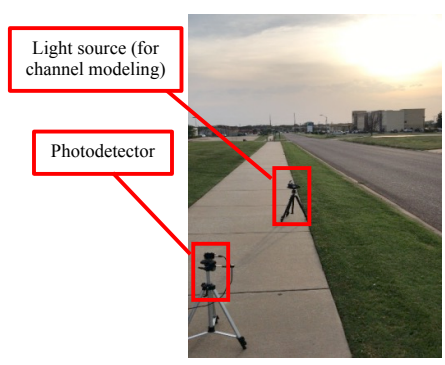

(a)

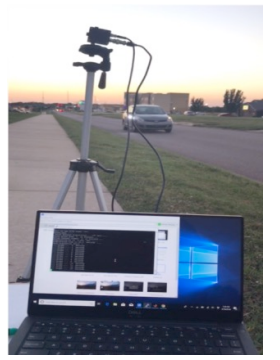

(b)
Fig. 2. A prototype for ViLDAR, a) road scenario under consideration and b) ViLDAR setup.

\subsection{Proposed algorithm}

In a companion paper 9, the authors discussed the channel model used to estimate vehicle speed based on the change in the received power from the vehicle's headlight. Fig. 3 depicts the algorithm used to calculate the vehicle speed from the collected data at the PD.

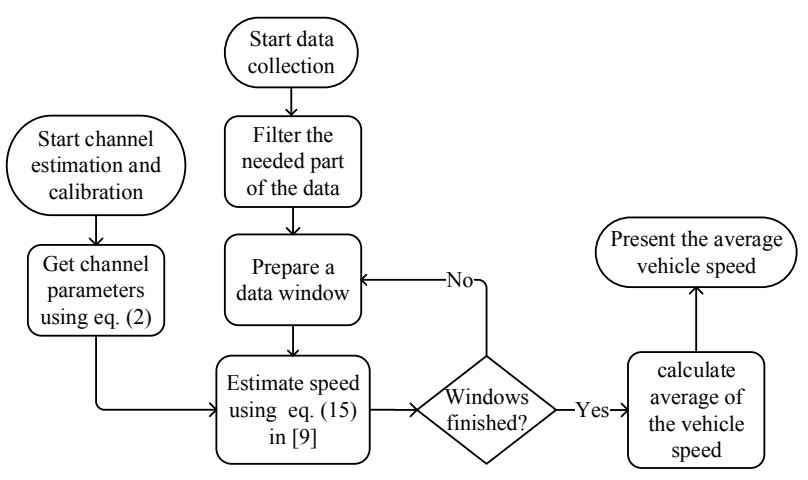

Fig. 3. Speed estimation algorithm.

The simplified model of the signal power associated with the visible light received by the PD at distance $D$ is given by:

$$
P_{r}=K \mathrm{D}^{-\gamma}
$$

where $D$ is the distance between transmitter (vehicle headlamp) and receiver (PD) in meters, $K$ is a constant that depends on the PD settings (area, angle, and field-ofview), $Y$ is the path-loss exponent which depends on the environmental conditions (reflectiveness of materials, humidity, etc.). Typically, the range of path-loss exponent lies between 1 and 5. As the vehicle approaches the PD the received light power increases. Notice also that for any values of $K$ and $Y$, the received power will change with the distance.

Equation (1) is used to estimate the values of $K$ and $y$ (referred to as the channel model parameters) and calibrate the ViLDAR system based on the prevailing environmental conditions. Measurements of the light intensity received by the PD are taken at known incremental distances $\left(D_{1}, D_{2}, \ldots, D_{n}\right)$ between the PD and light source in the range 3 to 15 meters. Least square (LS) estimation is applied to determine the values of the channel model parameters as follows:

$$
\boldsymbol{b}=\left[\boldsymbol{A}^{T} \boldsymbol{A}\right]^{-1} \boldsymbol{A}^{T} \mathbf{y}
$$

where $\boldsymbol{A}=\left(\begin{array}{cc}1 & 10 \log _{10}\left(D_{1}\right) \\ 1 & 10 \log _{10}\left(D_{2}\right) \\ \cdot & \cdot \\ 1 & 10 \log _{10}\left(D_{n}\right)\end{array}\right), \boldsymbol{b}=\left(\begin{array}{l}K \\ \gamma\end{array}\right)$ and $\boldsymbol{y}=\left(\begin{array}{c}P_{r}\left(D_{1}\right)_{d B} \\ P_{r}\left(D_{2}\right)_{d B} \\ \cdot \\ P_{r}\left(D_{n}\right)_{d B}\end{array}\right)$

The received power measurements are disaggregated for short time intervals (windows). Next, the raw data are filtered to reduce the measurement noise. Equation (15) in reference 9 is then used to estimate the speed for each window. Finally, the average vehicle speed estimation is calculated from the multiple windows estimates.

\section{Field measurements and results}

Preliminary data from the field experiments are presented in Error! Reference source not found.. The 
channel model parameters are estimated based on the measurements of the received signal power from the light source at the PD. In this experimental setting, $K$ was estimated to be $-49.5 \mathrm{~dB}$ and $y=1.18$ by using Equation (2).

In Fig. 4, the received light signal power of a moving vehicle is presented. As one can observe from Equation (1), when the vehicle approaches the PD (i.e., the distance decreases), the received signal increases. Once the vehicle passes the PD (i.e., outside the range of interest), the signal power reduces to the noise level.

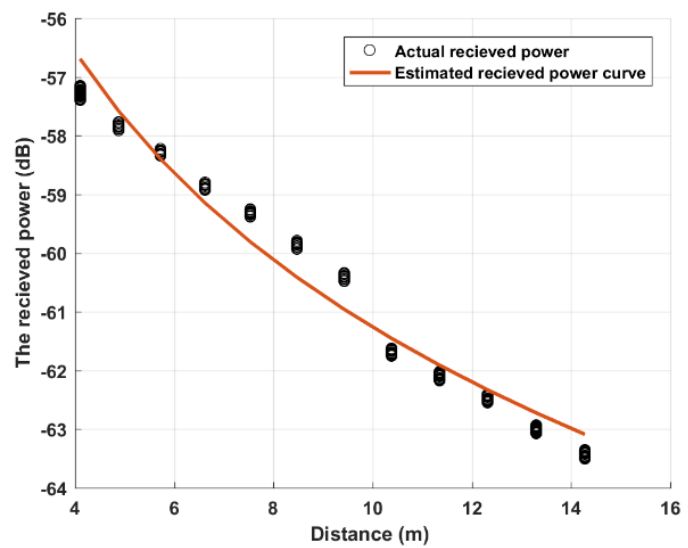

Fig. 5. Channel model estimation using LS.

\section{Preliminary conclusions}

In this work, the initial ViLDAR prototype and field measurement results are presented. More measurements will be taken in this project to optimize the algorithm and improve the speed estimation accuracy. The ViLDAR system performance will be evaluated under different scenarios and weather conditions.

\section{References}

1. Zheng, K., Zheng, Q., Chatzimisios, P., Xiang, W. and Zhou, Y. (2015). Heterogeneous Vehicular Networking: A Survey on Architecture, Challenges, and Solutions. IEEE Communications Surveys \& Tutorials, 17(4), 2377-2396, Fourth quarter 2015.

2. Fisher, P.D. (1992). Improving on police radar. IEEE Spectrum, 29(7), 38-43.

3. Turan, B., Narmanlioglu, O., Ergen, S.C., and Uysal, M. (2016). Physical Layer Implementation of Standard Compliant Vehicular VLC. IEEE 84th Vehicular Technology Conference (VTC-Fall), Sept 2016, 1-5.

4. Suzuki, A. J. and Mizui, K. (2015). Laser radar and visible light in a bidirectional V2V communication and ranging system. IEEE International Conference on Vehicular Electronics and Safety (ICVES), 19-24.

5. Cilean, A. M. and Dimian, M. (2017). Current challenges for visible light communications usage in vehicle applications: A survey. IEEE Communications Surveys Tutorials, 19(4), 26812703.
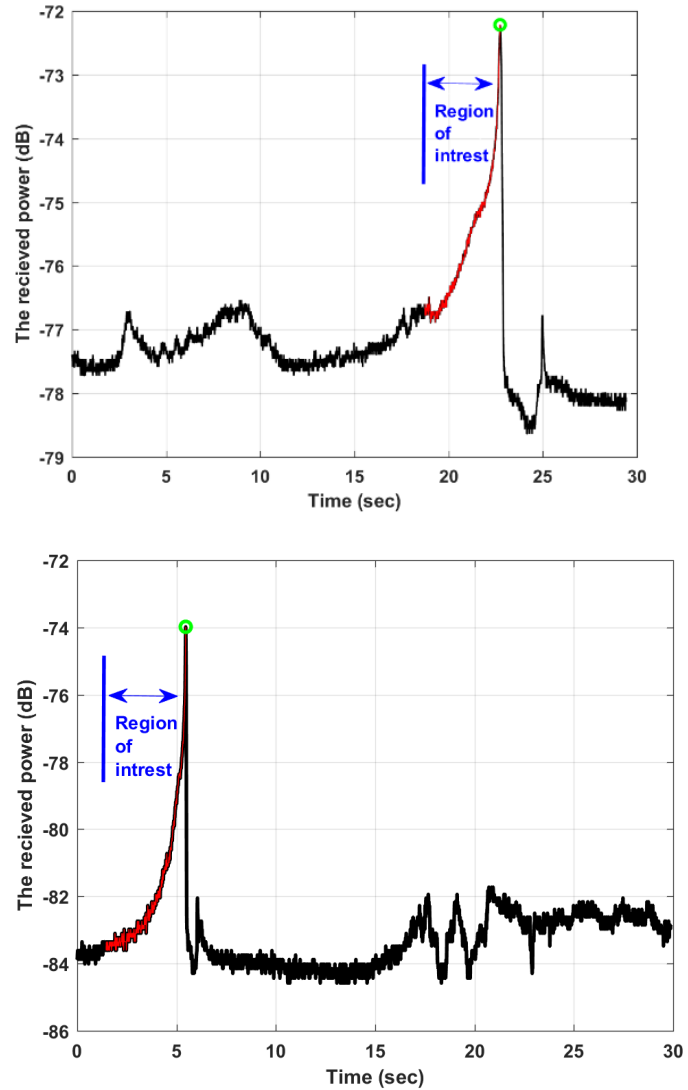

Fig. 4. ViLDAR measurement for different vehicle speeds of 5 $\mathrm{mph}$ (top) and $30 \mathrm{mph}$ (bottom).

6. Ucar, S., Ergen, S.C., and Ozkasap, O. (2018). IEEE 802.11p and Visible Light Hybrid Communication based secure autonomous platoon. IEEE Transactions on Vehicular Technology.

7. Cheng, L., Viriyasitavat, W., Boban, M., and Tsai, H.M. (2018). Comparison of radio frequency and visible light propagation channels for vehicular communications. IEEE Access, 6, 2634-2644.

8. Abuella, H., Ekin, S., Uysal, M. System and Method for Speed Estimation, Detection and Ranging using Visible Light in Vehicles. U.S. Provisional Patent Application No. 62/541,913.

9. Abuella. H., Miramirkhani, F., Ekin, S., Uysal, M., and Ahmed, S. ViLDAR - Visible Light Sensing Based Speed Estimation using Vehicle's Headlamps. Submitted to IEEE Transactions on Vehicular Technology.

10. Courtney, R.L. (1997). A broad view of ITS standards in the US. in Proceedings of Conference on Intelligent Transportation Systems, 529-536. 